\title{
The development of an accurate electronic aphid counter
}

\author{
P. G. F. Ploegaert
}

Technical and Physical Engineering Research Service (TFDL), Wageningen, the Netherlands

Accepted: 26 March 1976

Key words: electronic counter, aphid.

\section{Summary}

With the aid of an electronic counter a measuring instrument has been developed to count the number of aphids in a water suspension. The counting transducer consists of a photoelectric transistor with an active light beam which is intercepted by passing aphids. Without taking special precautions, accuracies of about $1 \%$ were obtained. This type of apparatus could be modified to count similar objects such as mites and the electronic circuit could be extended to obtain information about size division of colonies of aphids.

\section{Introduction}

To estimate the damage that aphids can cause to vegetation, the aphids from a number of sample plants in a test field are collected and counted. The count must be very accurate to obtain a reliable estimate for the whole field. Hand sorting, volumetric methods (Banks, 1954), photography (Hughes \& Woolcock, 1963) and electronic counters (Lowe \& Dromgoole, 1958; Hughes \& Woolcock, 1963) have all been tried. None of these methods have proved very accurate. Heathcote (1972) claims an accuracy of $\pm 6 \%$ for counts made on a grid and $\pm 3 \%$ with electronic counters, however several special precautions have to be made.

The apparatus described below developed for the Department of Entomology of the Agricultural University in Wageningen, gave more accurate countings of Aphis fabae, without special precautions.

\section{Apparatus and electronic measuring head}

A diagram of the measuring apparatus is given in Fig. 1. The funnel on the left is filled with a 1 litre suspension of about 4000 aphids in water and the exit closed. During counting this suspension must be stirred so that the aphids are evenly distributed throughout the water. With a difference of $25 \mathrm{~cm}$ between the level in the funnel and at the outlet, the current velocity is 0.25 litre per minute. During the 


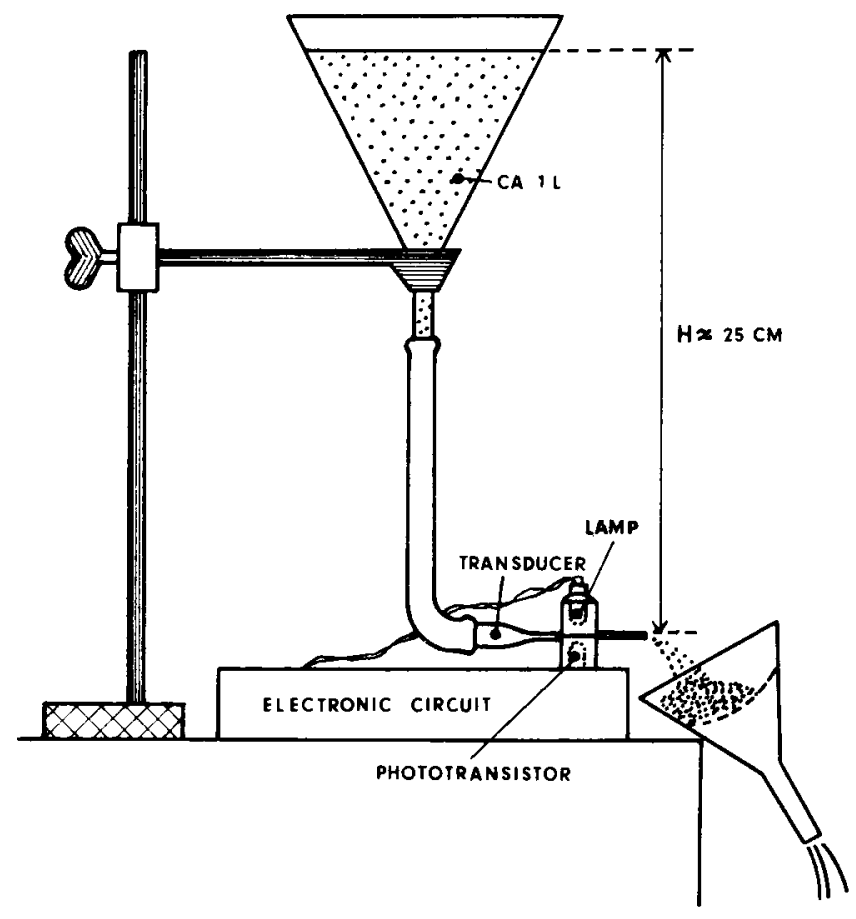

Fig. 1. Measuring system.

count the aphids pass through the measuring head which is shown in Fig. 2a and $2 \mathrm{~b}$. The end of the small brass tube has a $6 \mathrm{~mm}$ diameter opening, contracting to $2.8 \mathrm{~mm}, 1 \mathrm{~cm}$ from the outlet.

At one end the tube was shaped into a square pipe with internal breadth of $1.5 \mathrm{~mm}$ (Fig. 2a). In the square pipe, two Plexiglass windows, also with a breadth of $1.5 \mathrm{~mm}$, are positioned opposite each other. In a brass pipe a small lamp is placed opposite one window, and opposite the other window, a brass pipe with a light sensitive transistor OCP 71. To prevent unwanted reflections, the inside of the pipes are blackened. In the narrowing part of the transducer tube, the velocity of the water stream during the count increases and the aphids pass one by one between the window. Every aphid that passes is translated by the lamp and phototransistor combination into an electric pulse that is recorded on an electronic counter. A stoppage can be cleaned by putting a small square bar into the suare opening. Usually the error so incurred is only one count.

In Fig. 3, a block scheme is given of the electronic circuit. The electronic pulses are first spectrally filtered, then amplified and finally formed into a shape that can be easily counted by an electronic counter. 


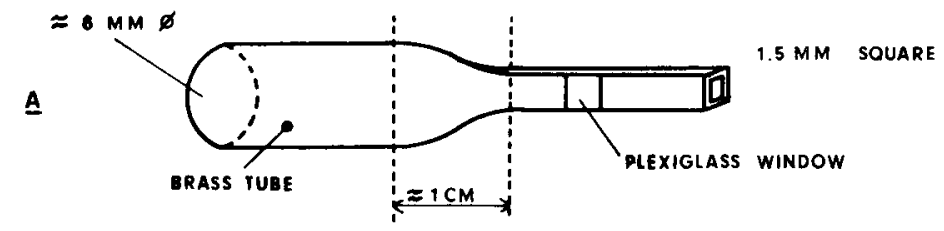

$\underline{\mathbf{B}}$

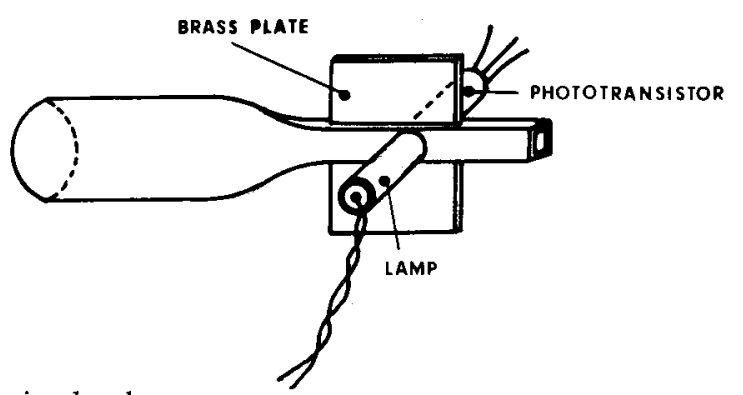

Fig. 2. Measuring head.

\section{Result of counts}

Measurements in the electronic laboratory

In the electronic laboratory, experiments were carried out on samples containing known numbers of large and small aphids. These samples were counted at different sensitivity levels of the electronic circuit. The knob of the sensitivity potentiometer has a scale from 1 to 4 .

In a sample 58 very small particles were counted by hand. The electronic count of this sample with the potentiometer at 1 , maximum sensitivity, always gave a result of 13 counts higher. After careful investigation of the sample 13 very small particles of dirt were found. With a filter with lower highfrequency cut-off the same results were obtained, confirming that in most cases the ground frequency determines the count. Decreasing the sensitivity (at higher settings) produced more variable results, because some particles were counted on some occasions and not on others, depending on how they were orientated as they passed the light beam (Table 1). Comparing the dimensions of these particles with the smallest aphids of a colony, a sensitivity setting in position 3 was chosen as the best general setting for counting aphids.

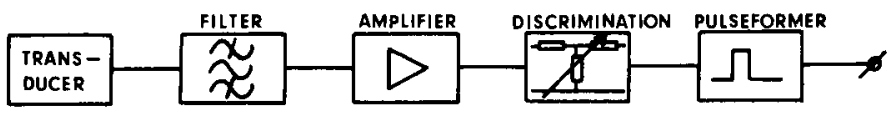

Fig. 3. Blockscheme of the circuit. 


\section{DEVELOPMENT OF AN ELECTRONIC APHID COUNTER}

Table 1. Effect of sensitivity setting on the variability of counts.

\begin{tabular}{ll} 
Sensitivity adjustment & Result of counts \\
1 & 58 \\
2 & $49-41$ \\
3 & $25-21$ \\
4 & $18-13$ \\
\hline
\end{tabular}

The following three measurements were made with the sensitivity setting in position 3. A series of preliminary counts produced the following results:

Sample with 287 aphids, counted by hand. The counts from the electronic counter were: $286,291,292,291,298,298,302$. The value increase with successive counts because the apparatus counted fragments that had broken of the aphids as they were handled, especially in older samples.

Sample containing 1375 aphids of different sizes viz.: 289 aphids $0.5-1 \mathrm{~mm}$; 383 aphids $0.2-0.5 \mathrm{~mm} ; 703$ aphids within $0.2 \mathrm{~mm}$. Four recorded counts were: $1440,1459,1457,1448$.

Sample containing about 2526 aphids, partial counted by hand and further estimated. The suspension volume was $0.5 \mathrm{l}$. The sample contained two big aphids that blocked the stream in the transducer. After removing these two the results were: $2597,2615,2617,2626$ and 2641.

\section{Results of counts at the Department of Entomology}

The apparatus was tested further at the Department of Entomology by Dr Ir G. W. Ankersmit, van de Bos and Rijsdijk. Measurements were made with a difference in level between funnel and outlet of $50 \mathrm{~cm}$ increasing the counting velocity, to cope with 1000 aphids a minute. When the density of the aphid suspension exeeded this, too few aphids were counted, probably because there were many coincidental passings. The reproducibility of the counts of samples with small, medium and large aphids is shown in Table 2. A drawback of the apparatus is that it cannot distinguish between aphids and fragments of dirt.

Table 2. Example of the reproducability of counts. Results are averages of 10 samples a, b and $c$.

$\begin{array}{lcl}\begin{array}{l}\text { Number of aphids } \\ \text { in the sample }\end{array} & \begin{array}{l}\text { Average number } \\ \text { of aphids }\end{array} & \begin{array}{l}\text { Standard } \\ \text { deviation }\end{array} \\ & 866 & \pm 17=1.9 \% \\ \text { a) } 0-2000 & 3352 & \pm 52=1.6 \% \\ \text { b) } 2000-5000 & 12025 & \pm 99=0.8 \% \\ \text { c) } 5000- & \end{array}$




\section{Future research}

When an aphid passes, a smaller or greater part of the active light beam will be intercepted dependent on the insect size. A bigger aphid gives a larger electronic pulse of longer duration. On this principle, discrimination circuits could be developed to provide information about the size division of colonies of aphids. This type of apparatus could also be used to count similar objects such as mites and eelworm cysts.

\section{Appendix}

\section{The electronic circuit}

Fig. 4 gives the electronic circuit, which was developed just before integrated circuits became commercially available. Eventhough the older type of components were used, good results were obtained.

The electronic filter. The stream velocity of the suspension between the Plexiglass

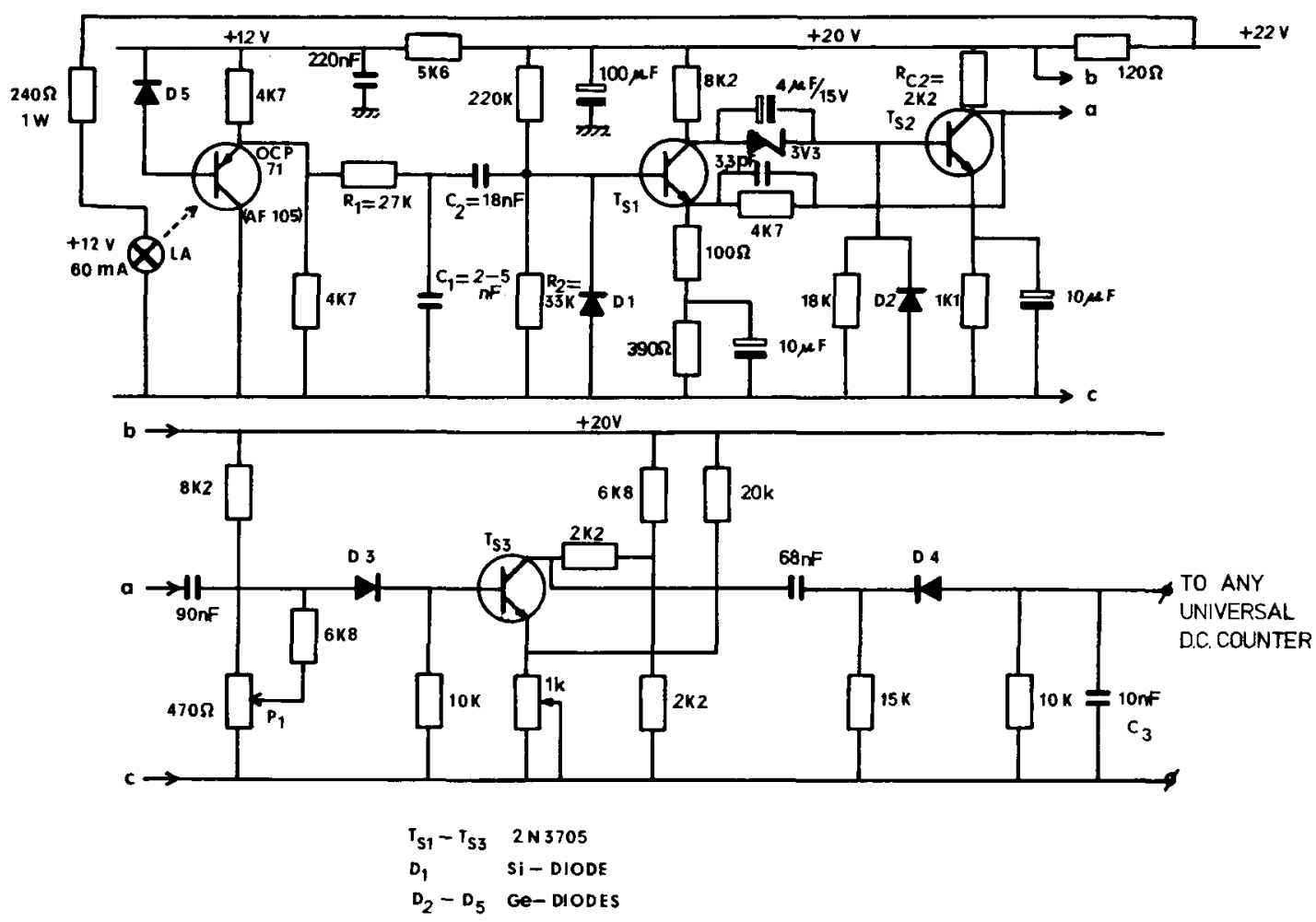

Fig. 4. Electronic circuit. 


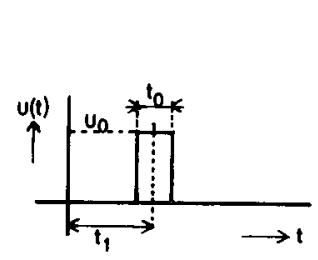

A

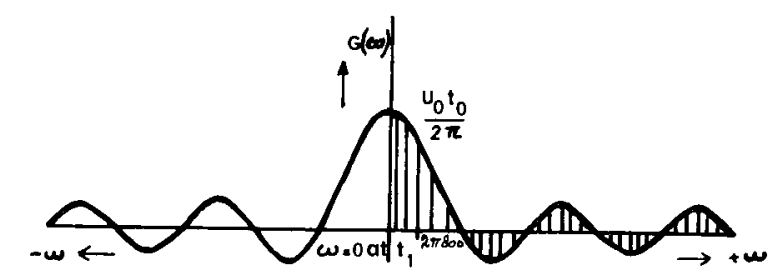

- THE SPECTRAL ENERgY division OF the SINGLE PULSE

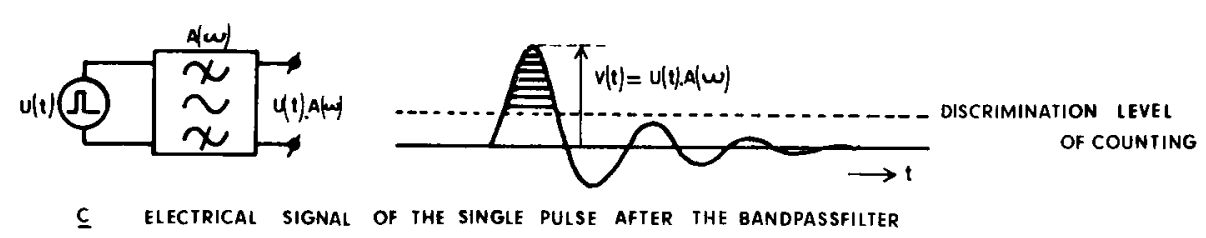

Fig. 5. Result of a single pulse passing through a bandpassfilter $\mathbf{A}(\psi)$.

windows is about $1.5 \mathrm{~m} / \mathrm{s}$. To calculate the filter we have to suppose two extreme situations. The upper limit is when aphids of $0.1 \mathrm{~mm}$ long flow through the tube immediately after each other. Here ground frequencies of up to $15 \mathrm{KHz}$ are produced. The lower limit occurs when a single aphid passes. A short pulse is produced with a spectral division from low to high frequencies. Fortunately a part of this spectrum is sufficient for a countable pulse, so a band-pass filter was fitted that rejects the lower and higher frequencies where the most faulty signals occur, e.g. the $50 \mathrm{~Hz}$ mains frequency. The spectral function caused by an aphid passing with a frequency of $50 \mathrm{~Hz}$ or less can still be counted, although the damping for the $50 \mathrm{~Hz}$ ground frequency is relatively great. The mains frequency is a sine wave without higher frequencies. Even when a single aphid passes, that produces a narrower pulse for a smaller aphid, it is counted, because the spectral energy division moves to higher frequencies, that lie in the pass-band. Thus available energy as function of the aphid size has a sort of feedback.

Fig. 5 shows the treatment of a single pulse (Guillemin, 1958). The spectral energy division of the pulse, $G(\omega)$ in Fig. $5 b$ has the formula:

$$
G(\omega)=\frac{t_{o}}{2 \pi} \cdot U_{o} \varepsilon t_{1}^{j} \cdot \frac{\sin \omega \frac{t_{o}}{2}}{\omega \frac{t_{o}}{2}} .
$$

The result after passing through the band filter is shown in Fig. $5 \mathrm{c}$. The shaded part is countable.

The band-pass filter is of the RC-CR type which has a broad pass-band that can be varied, for instance with $\mathrm{C} 1$ (Fig. 6). 


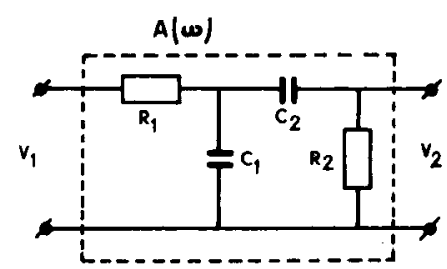

Fig. 6. The bandpassfilter.

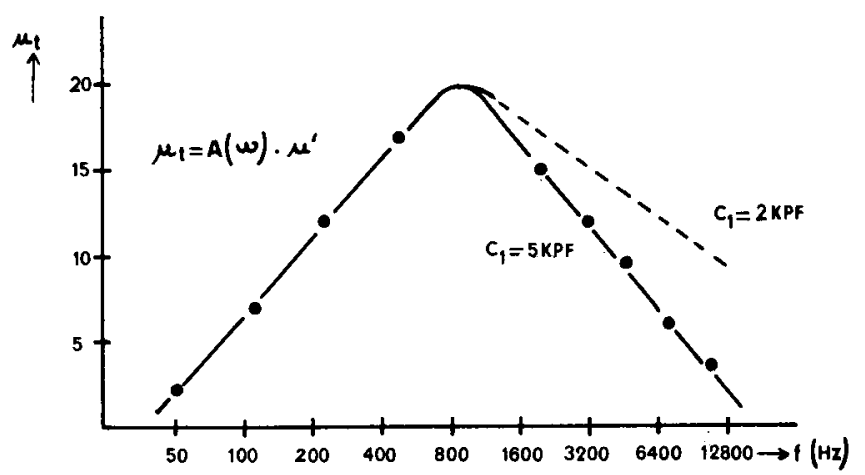

Fig. 7. Total transfer $\mu_{1}$ of the combination bandfilter and amplifier as a function of the frequency.

The amplifier. The pulses coming from the filter are amplified about 50 times. The input impedance is about $75 \mathrm{k} \Omega$, the output impedance about $150 \Omega$ due to the feedback resistance of $4.7 \mathrm{k} \Omega$. Fig. 7 shows the total transfer function of the combination band filter and amplifier as a function of the frequency.

Sensitivity adjustment. The sensitivity of the circuit can be adjusted with the discrimination level potentiometer $\mathrm{P}_{1}$, producing levels from $0.1 \mathrm{~V}$ to $0.8 \mathrm{~V}$. With maximum sensitivity of the circuit, an $1 \mathrm{mV}$ input pulse gives a $2 \mathrm{~V}$ output pulse. The optimum discrimination level adjustment has to be determined experimentally, so that disturbances and secondary pulses are suppressed, at least $6 \mathrm{~dB}$ (Fig. 5c).

End pulse former. Finally the pulses are amplified with $\mathrm{Ts}_{3}$ and again protected against unwanted transients with $\mathrm{D}_{4}$ and $\mathrm{C}_{3}$.

\section{References}

Lowe, A. D. \& W. V. Dromgoole, 1958. Electronic aphid counter. N.Z. Jl agric. Res. 1: No 6. Hughes, R. D. \& L. T. Woolcock, 1963. Electronic counter for cabbage aphid. N.Z. Jl agric. Res. 6: No 3 and 4.

Heathcote, G. D., 1972. In: H. F. van Emden (Ed.), Evaluating aphid populations or plants in aphid ttechnology. Acad. Press, London/New York, pp. 105.145.

Banks, C. J., 1954. A method for estimating populations and counting large number of Aphis fabae. Scop. Bull. ent. Res. 45: 751-756.

Guillemin, E. A., 1958. The mathematics of circuit analysis. John Wiley \& Sons, New York and Chapman \& Hall Ltd., p. 472. 